\title{
ADAPTIVE VISUAL-FORCE CONTROL IN UNKNOWN WORKSPACES
}

\author{
Jorge Pomares, Fernando Torres, Laura Payá \\ Physics, Systems Engineering and Signal Theory Department \\ University of Alicante, Alicante, Spain \\ Email: \{jpomares,Fernando.Torres,laura.paya\}@ua.es
}

\begin{abstract}
Keywords: force control, image-based control, autocalibration.
Abstract: $\quad$ This paper proposes the definition of a new adaptive system that combines visual and force information. At each moment, the proportion of information used from each sensor is variable depending on the adequacy of each sensor to control the task. The sensorial information obtained is processed to allow the use of both sensors for controlling the robot and avoiding situations in which the control actions are contradictory. Although the visual servoing systems have certain robustness with respect to calibration errors, when the image-based control systems are combined with force control we must accurately know the intrinsic parameters. For this purpose an adaptive approach is proposed which updates the intrinsic parameters during the task.
\end{abstract}

\section{INTRODUCTION}

Image-based visual servoing is now a well-known approach for positioning the robot with respect to an object observed by a camera mounted at the robot end-effector (Hutchinson et al. 1996). However, in applications in which the robot must interact with the workspace, the visual information must be combined with the sensorial information obtained from the force sensor. A great number of approaches employed for fusing the information obtained from both sensors have been based, up to now, on hybrid control. Concerning hybrid visual-force systems, we should mention studies like (Baeten and De Schutter, 2002) which extend the "task frame" formalism (Bruyninckx and De Schutter, 1996). In (Namiki et al., 1999) a system for grasping objects in real time, which employs information from an external camera and that obtained from the force sensors of a robotic hand, is described. Another strategy used for the combination of both sensorial systems is the use of impedance control. Based on the basic scheme of impedance control, we should mention several modifications like the one described in (Morel et al., 1998), which adds an external control loop that consists of a visual controller which generates the references for an impedance control system. In works such as (Tsuji et al. 1997), the use of virtual forces applied to approaching tasks without contact, is proposed.

In this paper we are not interested in image processing issues, so that the tracked target is composed of four grey marks which will be the extracted features during the tracking. This paper proposes the definition of a new adaptive system which combines visual and force information. Similar approaches has been developed in works such as (Baeten et al., 2002; Olson et al. 2002) however these approaches do not consider the possibility of both sensors providing contradictory information at a given moment of the task. Thus, in unstructured environments it can happen that the visual servoing system establishes a movement direction that is impossible according to the interaction information obtained from the force sensor. In this paper, we consider this possibility and the sensory information obtained is processed to allow the use of both sensors for controlling the robot.

An original aspect of the proposed system is that the proportion of information used from each sensor is variable and depends on the criterion described in Section 4. At each moment, this criterion provides information about the sensor more adequate to develop the task. 
This paper is organized as follows: The main characteristics of the trajectory to be tracked and the notation used is described in Section 2. Section 3 shows the way in which the tracking of the trajectory in the image is carried out. In Section 4, the strategy used for fusing force information with that from the visual servoing system is described. Section 5 describes how the fusion system manages situations in which contradictory control actions are obtained from both sensorial systems. The autocalibration system employed to update the intrinsic parameters is described in Section 6. In Section 7, experimental results, using an eye-in-hand camera, confirm the validity of the proposed algorithms. The final section presents the main conclusions arrived at.

\section{NOTATION}

In this paper, the presence of a planner, which provides the robot with the 3-D trajectory, $\gamma(\mathrm{t})$, to be tracked (i.e., the desired 3-D trajectory of the camera at the end-effector), is assumed. These trajectories are generated from a 3-D geometric model of the workspace, so that it is necessary to employ a visual servoing system that performs the tracking of the 3$\mathrm{D}$ trajectory using visual information and, at the same time, tests whether it is possible to carry out such tracking, depending on the interaction forces obtained.

By sampling $\gamma(\mathrm{t})$ (with period $T$ ), a sequence of $\mathrm{N}$ discrete values is obtained, each of which represents $\mathrm{N}$ intermediate positions of the camera ${ }^{\mathrm{k}} \gamma / \mathrm{k} \in 1 \ldots \mathrm{N}$. From this sequence, the discrete trajectory of the object in the image $S=\left\{{ }^{k} \mathbf{s} / k \in 1 \ldots N\right\}$ can be obtained, where ${ }^{\mathrm{k}} \mathbf{s}$ is the set of $\mathrm{M}$ point or features observed by the camera at instant $\mathrm{k}$, ${ }^{\mathrm{k}} \mathbf{s}=\left\{{ }^{\mathrm{k}} \boldsymbol{f}_{\mathrm{i}} / \mathrm{i} \in 1 \ldots \mathrm{M}\right\}$. As we have previously indicated, in this paper we are not interested in image processing issues, therefore, the tracked target is composed of four grey marks whose centres of gravity will be the extracted features (see Section 7).

The following notations are used. The commanded velocity for the visual servoing and for the force control systems are $\boldsymbol{v}_{\mathrm{V}}^{\mathrm{C}}$ and $\boldsymbol{v}_{\mathrm{F}}^{\mathrm{C}}$ respectively. $\boldsymbol{F}\left(f_{\mathrm{x}}, f_{\mathrm{y}}, f_{\mathrm{z}}, n_{\mathrm{x}}, n_{\mathrm{y}}, n_{\mathrm{z}}\right)$ are force $(\mathrm{N})$ and torque $(\mathrm{N} \mathrm{m})$ exerted by the environment onto the robot and $k$ is the tool stiffness ( $\mathrm{N} \mathrm{m}$ or $\mathrm{N} \mathrm{m} \mathrm{rad}{ }^{-1}$ ). $\lambda_{\mathrm{V}}$ and $\lambda_{\mathrm{F}}$ are the proportional control gains for the visual and force controllers respectively.

\section{VISUAL TRACKING OF TRAJECTORIES}

Each sample, ${ }^{\mathrm{k}} \mathbf{s}$, is generated from each position ${ }^{\mathrm{k}} \gamma$. These positions are obtained considering that the time between two consecutive samples is constant, so that $\Delta \Delta^{\mathrm{k}+1} t={ }^{\mathrm{k}+1} t-{ }^{\mathrm{k}} t=T$ where $T$ is the video rate. The desired trajectory to be tracked in the image is obtained using a natural cubic B-spline (the spline interpolation problem is states as: given image points $\mathrm{S}=\left\{{ }^{\mathrm{k}} \mathrm{s} / \mathrm{k} \in 1 \ldots \mathrm{N}\right\}$ and a set of parameter values $\Gamma=\left\{{ }^{k} t / \mathrm{k} \in 1 \ldots \mathrm{N}\right\}$ we want a cubic B-spline curve $\mathbf{s}(t)$ such that $\left.\mathbf{s}\left(t_{\mathrm{k}}\right)={ }^{\mathrm{k}} \mathbf{s}\right)$ :

$$
\mathbf{s}_{\mathrm{d}}(t)={ }^{\mathrm{k}} \mathbf{A} t^{3}+{ }^{\mathrm{k}} \mathbf{B} t^{2}+{ }^{\mathrm{k}} \mathbf{C} t+{ }^{\mathrm{k}} \mathbf{D}
$$

where ${ }^{\mathrm{k}} \mathbf{A},{ }^{\mathrm{k}} \mathbf{B},{ }^{\mathrm{k}} \mathbf{C},{ }^{\mathrm{k}} \mathbf{D}$ are obtained from the samples in the image space at the given instants. To perform the tracking of the desired trajectory in the image space, an image-based control scheme to regulate to 0 the following vision-based task function is used (Mezouar and Chaumette, 2002):

$$
\boldsymbol{e}=\hat{\mathbf{J}}_{\mathrm{f}}^{+} \cdot\left(\mathbf{s}-\mathbf{s}_{\mathrm{d}}(t)\right)
$$

where $\mathbf{s}$ are the extracted features from the image and $\hat{\mathbf{J}}_{\mathrm{f}}^{+}$is an estimation of the pseudoinverse of the interaction matrix. To carry out the tracking of the trajectory, the following velocity must be applied to the robot (with respect to the coordinate frame located at the eye-in-hand camera):

$$
\boldsymbol{v}_{\mathrm{V}}^{\mathrm{C}}=-\lambda_{\mathrm{V}} \cdot \boldsymbol{e}+\widehat{\mathbf{J}}_{\mathrm{f}}^{+} \cdot \frac{\partial \mathbf{s}_{\mathrm{d}}(t)}{\partial t}
$$

where $\lambda_{\mathrm{V}}>0$ is the gain of the proportional controller.

\section{FUSION VISUAL-FORCE CONTROL}

Up to now, the majority of approaches for fusing visual and force information are based on hybrid control. Only recently (Baeten et al., 2002) has it been possible to find studies on the control of a given direction using force and vision simultaneously (shared control). These approaches are based on the "task frame" formalism 
(Bruyninckx and De Schutter, 1996). These works suppose the presence of a high level descriptor of the actions to be carried out in each direction of the work-space at each moment of the task. Thus, the geometric properties of the environment must be known previously. The approach described in this section does not require specifying the sensorial systems to be used for each direction. Furthermore, the proportion of information used from each sensor depends on the criterion described in this section.

The GLR algorithm (Generalized Likelihood Ratio) (Willsky and Jones, 1976) applied to the obtained forces is employed for fusing visual and force information (the setup of the different parameters of the GLR can be seen in our previous works (Pomares and Torres, 2005)). If a given task consists of using visual and force information for maintaining a constant contact with a surface, when the value of GLR increases, this can obtained when, for several possible reasons (irregularities in the surface, errors in the trajectory generated by the visual servoing system, high velocity, etc.) the tracking is not correctly done and, therefore, the system cannot maintain a constant force on the surface. The behaviour is then more oscillatory, and changes are generated in the interaction forces, increasing the value of GLR. To correct this behaviour, the proportion of information used from the force sensor can be augmented when the value of GLR increases, as described below.

The final control action, $\boldsymbol{v}^{\mathrm{C}}$, will be a weighted sum obtained from the visual servoing system, $\boldsymbol{v}_{\mathrm{V}}^{\mathrm{C}}$, and from the force sensor, $\boldsymbol{v}_{\mathrm{F}}^{\mathrm{C}}=\boldsymbol{\lambda}_{\mathrm{F}} \cdot\left(\boldsymbol{F}-\boldsymbol{F}_{\mathrm{d}}\right) / k$, so that $\boldsymbol{v}^{\mathrm{C}}=p_{\mathrm{V}} \cdot \boldsymbol{v}_{\mathrm{V}}^{\mathrm{C}}+p_{\mathrm{F}} \cdot \boldsymbol{v}_{\mathrm{F}}^{\mathrm{C}}$. Depending on the value of GLR, we obtain the following control actions:

$\underline{\mathrm{GLR}<\mathrm{U}_{1}}$. Normal functioning of the system. In this case, both control actions are weighted with the same proportion (empirically $\mathrm{U}_{1}=500$ is obtained):

$$
\boldsymbol{v}^{\mathrm{C}}=0,5 \cdot \boldsymbol{v}_{\mathrm{V}}^{\mathrm{C}}+0,5 \cdot \boldsymbol{v}_{\mathrm{F}}^{\mathrm{C}}
$$

$\underline{\mathrm{U}}_{1} \leq \mathrm{GLR}<\mathrm{U}_{2}$. Range of values of GLR that can be obtained when a change in the surface begins or when the system works incorrectly (empirically $\mathrm{U}_{2}=1000$ ). In this case, the weight applied to the control action corresponding to the visual servoing system is reduced with the aim of correcting defects in the tracking. Before describing the weight function for this range of GLR, two parameters that characterize this function, are defined. These parameters $\left(p_{1}, p_{2}\right)$ identify the velocity range that the visual servoing system can establish for different values of GLR. Thus, when GLR is equal to $\mathrm{U}_{1}$, or lower, the velocity established by the computer vision system will be $\boldsymbol{v}_{\mathrm{V} \max }^{\mathrm{C}}=-\frac{\lambda_{\mathrm{V}}}{2} \cdot \boldsymbol{e}+\widehat{\mathbf{J}}_{\mathrm{f}}^{+} \cdot \frac{\partial \mathbf{s}_{\mathrm{d}}(t)}{\partial t}$, that is to say, the normal velocity defined to carry out the tracking of the trajectory in the image space. In the previous expression, we can see the term $\lambda_{\mathrm{V}} / 2$ due to the weight in the control action obtained from the computer vision system, $\boldsymbol{v}_{\mathrm{V}}^{\mathrm{C}}$, in the global control action, $\boldsymbol{v}^{\mathrm{C}}$, that is to say, $p_{1}=0,5$ (see Equation (4)). However, when GLR is equal to $\mathrm{U}_{2}$, we define $\boldsymbol{v}_{\mathrm{V} \text { min }}^{\mathrm{C}}=-\lambda_{\mathrm{V}} \cdot p_{2} \cdot \boldsymbol{e}+\widehat{\mathbf{J}}_{\mathrm{f}}^{+} \cdot \frac{\partial \mathbf{s}_{\mathrm{d}}(t)}{\partial t}<\boldsymbol{v}_{\mathrm{V} \text { max }}^{\mathrm{C}}$ as the minimum velocity, empirically obtained, to carry out the tracking of the trajectory and which allows the system to correct the possible defects in this trajectory (the effect of the force control in the trajectory is increased in the global control action). Thus, the value of the weight associated with the velocity provided by the visual servoing system, will be $p_{2}=0,5 \cdot \frac{\boldsymbol{v}_{\mathrm{V} \min }^{\mathrm{C}}}{\boldsymbol{v}_{\mathrm{V} \max }^{\mathrm{C}}}$. Therefore, considering a decreasing evolution of the weight function applied to the velocity obtained from the visual servoing system, this function will have the following value in the range $\mathrm{U}_{1} \leq \mathrm{GLR}<\mathrm{U}_{2}$ :

$$
p_{\mathrm{v}}=\frac{p_{2}-p_{1}}{\mathrm{U}_{2}-\mathrm{U}_{1}} \cdot \mathrm{GLR}+p_{1}-\frac{p_{2}-p_{1}}{\mathrm{U}_{2}-\mathrm{U}_{1}} \cdot \mathrm{U}_{1}
$$

Obviously, the weight associated with the force control system will be $p_{\mathrm{F}}=1-p_{\mathrm{v}}$.

GLR $>\mathrm{U}_{2}$. When GLR is in this range, the behaviour established is to continue with the minimum velocity, $\boldsymbol{v}_{\mathrm{V} \text { min }}^{\mathrm{C}}$.

\section{MANAGING CONTRADICTORY CONTROL ACTIONS}

Up to now, the approaches for fusing visual and force information do not consider the possibility of both sensors providing contradictory information at a given moment of the task (the visual servoing system establishes a movement direction that is impossible according to the interaction information obtained from the force sensor).

To assure that a given task in which it is required an interaction with the setting is correctly developed, the system must carry out a variation of the trajectory in the image, depending on the spatial restrictions imposed by the interaction forces. Therefore, given a collision with the setting and having recognized the normal vector of the contact 
surface (Pomares and Torres, 2005), the transformation $\mathbf{T}_{\mathrm{r}}$ that the camera must undergo to fulfil the spatial restrictions, is determined. This transformation is calculated so that it represents the nearest direction to the one obtained from the imagebased control system, and which is contained in the plane of the surface. Thus, we guarantee that the visual information will be coherent with the information obtained from the force sensor. To do so, considering $\boldsymbol{f}$ to be the position of a given feature extracted by the camera at a given instant, and $\left[\mathbf{R}_{\mathbf{i}} \mathbf{t}_{\mathbf{i}}\right]$ (rotation and translation) a sampling of the transformation $\mathbf{T}_{\mathrm{r}}$ that the camera undergoes during the tracking of the recognized surface, the feature $f_{\mathrm{i}}^{\prime}$ extracted in each one of these positions will be:

$$
\boldsymbol{f}_{\mathrm{i}}^{\prime}=\mathbf{A} \cdot \mathbf{R}_{\mathrm{i}} \cdot \mathbf{A}^{-1} \cdot \boldsymbol{f}+\mathbf{A} \cdot \mathbf{t}_{\mathrm{i}} / z
$$

where $\mathrm{z}$ is the distance between the camera and the object from which the features are extracted and $\mathbf{A}$ is the following intrinsic parameter matrix:

$$
\mathbf{A}=\left[\begin{array}{ccc}
\mathrm{f} \cdot p_{\mathrm{u}} & -\mathrm{f} \cdot p_{\mathrm{u}} \cdot \cot (\theta) & u_{0} \\
0 & \mathrm{f} \cdot p_{\mathrm{v}} / \sin (\theta) & v_{0} \\
0 & 0 & 1
\end{array}\right]
$$

Considering the homogeneous image coordinates of a feature $\boldsymbol{f}_{\mathrm{i}}=\left[u_{\mathrm{i}}, v_{\mathrm{i}}, 1\right], u_{0}$ and $v_{0}$ are the pixel coordinates of the principal point, $\mathrm{f}$ is the focal length, $p_{\mathrm{u}}$ and $p_{\mathrm{v}}$ are the magnifications in the $u$ and $v$ directions respectively, and $\theta$ is the angle between these axes.

From the sampling of the desired trajectory in the image, $\boldsymbol{f}_{\mathrm{i}}^{\prime}$, a spline interpolator is applied to obtain the desired trajectory in the image (see Section 3 ).

\section{AUTOCALIBRATION}

It is well known that the visual servoing systems have certain robustness with respect to calibration errors. However, the knowledge of the intrinsic parameters is important when visual and force information is combined, in order to deal with contradictory control actions obtained from both sensorial systems. As can be seen in (6) it is necessary to know A for determining the new trajectory in the image once the collision is detected. The matrix $\mathbf{A}$ is obtained by a previous calibration of the camera using the Zhang's method (Zhang, 2000). However, during the task the intrinsic and extrinsic parameters can be modified. In order to update the camera intrinsic and extrinsic parameters the following method is employed.

We assume that the focal length in $u$ and $v$ directions differ, denoting $f_{\mathrm{u}}, f_{\mathrm{v}}$ respectively. The estimated camera intrinsic parameters are $\mathrm{P}_{\mathrm{I}}=\left[f_{\mathrm{u}}, f_{\mathrm{v}}\right.$, $\left.u_{0}, v_{0}\right]$. At a given instant $\mathrm{k}$, using these parameters we obtain a set of features ${ }^{\mathrm{k}} \mathbf{s}_{\mathrm{I}}=\left\{{ }^{\mathrm{k}} \boldsymbol{f}_{\mathrm{Ii}} / \mathrm{i} \in 1 \ldots \mathrm{M}\right\}$. When the set $P_{I}$ varies, the derivative of $\mathbf{s}_{I}$ with respect to the change of the intrinsic parameters is:

$$
\dot{\mathbf{s}}_{\mathrm{I}}=\frac{\partial \mathbf{s}_{\mathrm{I}}}{\partial \mathrm{P}_{\mathrm{I}}} \cdot \frac{\partial \mathrm{P}_{\mathrm{I}}}{\partial t}
$$

Considering $\mathbf{s}$ the true features extracted from the image, the error function $\xi=\mathbf{s}-\mathbf{s}_{I}$ is defined. Therefore:

$$
\dot{\xi}=\mathbf{J}_{\mathrm{f}} \mathbf{T}+\frac{\partial \mathbf{s}_{\mathrm{I}}}{\partial \mathrm{P}_{\mathrm{I}}} \cdot \frac{\partial \mathrm{P}_{\mathrm{I}}}{\partial t}
$$

where $\mathbf{T}$ is the variation with respect the time of the extrinsic parameters, and $\mathbf{J}_{\mathrm{f}}$ the interaction matrix for four points (Marchand and Chaumette, 2002) corresponding to the four features.

As we have previously described, the intrinsic parameters must be known when a collision is detected. When $\xi$ is equal to 0 the intrinsic parameters, $\mathrm{P}_{\mathrm{I}}$, corresponds with the true ones. To make $\xi$ decrease exponentially to 0 we form the feedback loop to this system where the feedback value should be:

$$
\left[\begin{array}{c}
\mathbf{T} \\
\dot{\mathrm{P}}_{\mathrm{I}}
\end{array}\right]=-\mathrm{k}_{\mathrm{C}} \cdot\left[\begin{array}{ll}
\mathbf{J}_{\mathrm{f}} & -\frac{\partial \mathbf{s}_{\mathrm{I}}}{\partial \mathrm{P}_{\mathrm{I}}}
\end{array}\right]^{+} \cdot \xi
$$

Therefore, the extrinsic and intrinsic parameters must be determined when a collision occurs. To do so, we move the camera according to the $\mathbf{T}$ component and the intrinsics with $\mathrm{P}_{\mathrm{I}}=\mathrm{P}_{\mathrm{I}}+\dot{\mathrm{P}}_{\mathrm{I}}$ until $\xi$ is 0 . At this moment the true camera parameters will be know and the Equation (6) can be applied to obtain the new image trajectory which must be tracked.

\section{RESULTS}

In this section, we describe the different tests carried out that show the correct behaviour of the system. 
For the tests we have used an eye-in-hand camera system composed of a JAI-M536 mini-camera in the end-effector of a 7 d.o.f. Mitsubishi PA-10 robot also equipped with a force sensor (67M25A-I40 from JR3. Inc.). MATROX GENESIS is used as the image acquisition and processing board. The system is able to acquire up to 30 frames/second and is previously submitted to a calibration process (focal length is $7,5 \mathrm{~mm}$ ). In the experiments described in this paper, the tracked target is composed of four grey marks (see Figure 1).

Figure 1 shows the surface which the robot must track using visual-force control (we can observe that the surface presents a discontinuity). Applying the sensorial fusion algorithm described in Section 4, the evolution of the forces and of the GLR obtained from these forces is shown in Figure 2.

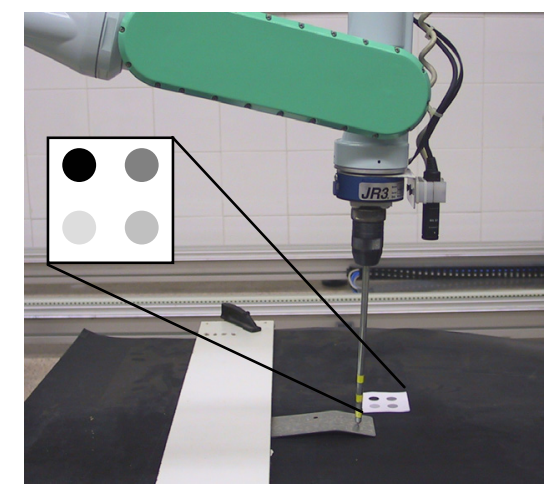

Figure 1: Experimental setup.
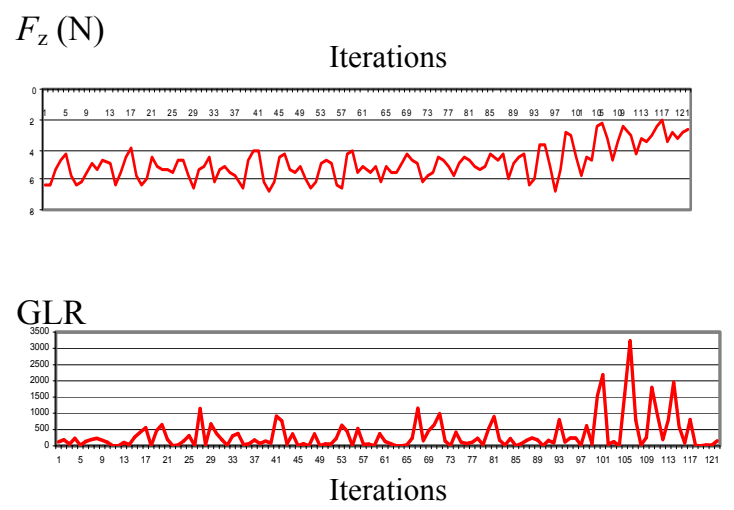

Figure 2: Evolution of the force and the corresponding GLR. Experiment 1 (convex surface).

Figure 2 show that GLR presents greater values when the robot is not able to maintain the constant contact with the surface. This fact can be observed in the discontinuity of the surface.
Figure 3 and Figure 4 show two experiments for tracking a plane surface. The first graph of each figure represents the applied force in $\mathrm{z}$ direction fusing visual and force information with constant weights. In the second graph the proposed strategy of variable weights is used (see Section 4). We can observe that using the strategy of variable weights the system response is less oscillating. Using this strategy the system allows maintaining the constant contact force with the surface.
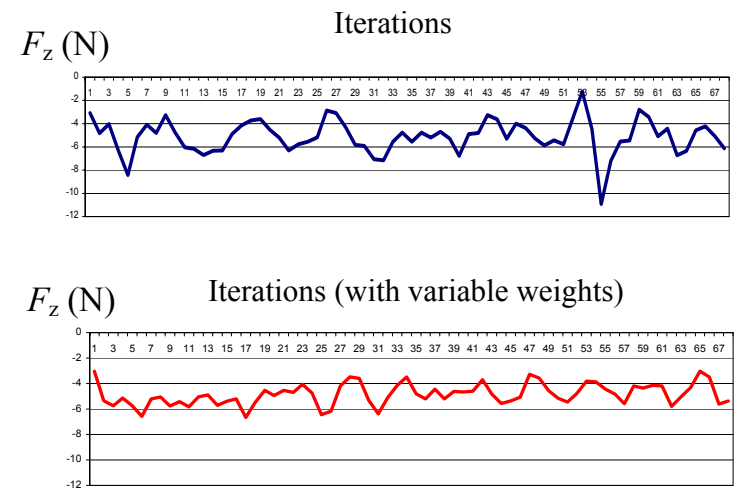

Figure 3: Comparison between the obtained forces without using and using the strategy of variable weights. Experiment 2.

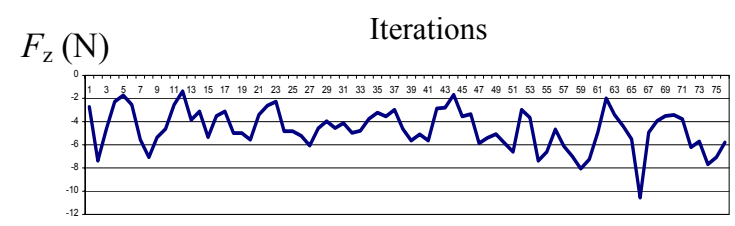

$F_{\mathrm{z}}(\mathrm{N}) \quad$ Iterations (with variable weights)

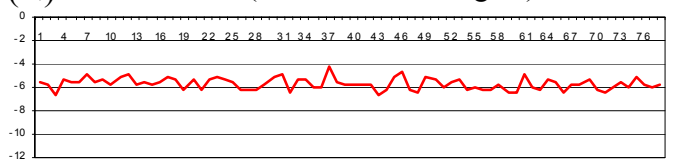

Figure 4: Comparison between the obtained forces without using and using the strategy of variable weights. Experiment 3.

When a collision is detected the system updates the intrinsic parameters to guarantee that the new trajectory is generated correctly. To illustrate the behaviour of the algorithm we show an autocalibration experiment. Figure 5 shows the image trajectory obtained varying the intrinsic parameters until $\xi=\mathbf{s}-\mathbf{s}_{\mathrm{I}}$ is zero. The convergence of the focal length estimations is shown in Figure 6 (the pixel is almost the same in $u$ and $v$ directions on the image sensor). 


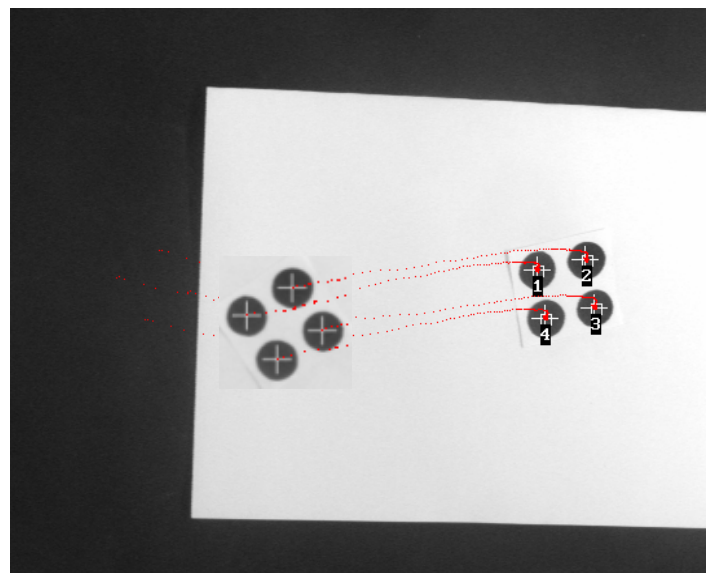

Figure 5: Image trajectory during the autocalibration.

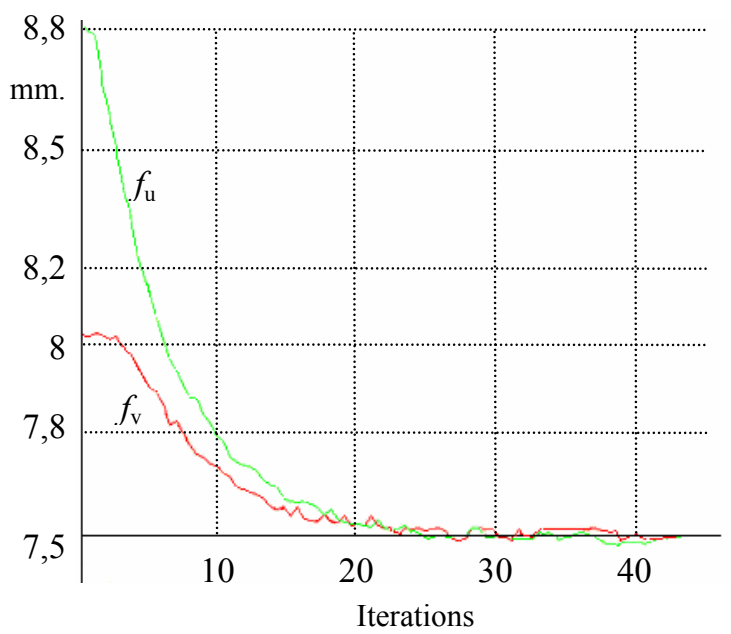

Figure 6: Convergence of the estimated focal lengths.

\section{CONCLUSIONS}

We proposed a new method for combining visual and force information which allow us to update the intrinsic parameters during the task by using an autocalibration approach. The visual-force control system has others original aspects which improve the behaviour of the system. Within these aspects we should mention the variable weights applied to each sensor (depending on the GLR parameter) and the possibility of managing contradictory control actions. As the results show, the robot is able to track the image trajectory maintaining a constant force with the workspace using visual and force information simultaneously.

\section{REFERENCES}

Baeten, J., De Schutter, J., 2002, Hybrid Vision/Force Control at Corners in Planar Robotic-Contour Following. IEEE/ASME Transactions on Mechatronics, vol. 7, no 2, pp. $143-151$.

Baeten, J., Bruyninckx, H., De Schutter, J, 2002. Shared Control in Hybrid Vision/Force robotic Servoing using the Task Frame. In Proceedings of the 2002 IEEE/RSJ International Conference on Intelligent Robots and Systems. Lausanne, Suiza. Pp. 2128-2133.

Bruyninckx, H., De Schutter, J., 1996. Specification of force-controlled actions in the task frame formalism-A synthesis, IEEE Transactions on Robotics and Automation, vol. 12, no. 4. pp. 581-589.

Hutchinson, S., Hager, G., Corke, P., 1996. A Tutorial on Visual Servo Control. IEEE Trans. on Robotics and Automation, vol. 12, no. 5, pp. 651-670.

Marchand, E., Chaumette, F., 2002. Virtual Visual Servoing: a framework for real-time augmented reality. In EUROGRAPHICS 2002 Conference Proceeding, Computer Graphics Forum, Sarrebruck, Germany. vol. 21, no. 3, pp. 289-298.

Mezouar, Y., Chaumette, F., 2002. Path Planning For Robust Image-based Control. IEEE Transactions on Robotics and Automation, Vol. 18, No. 4, pp. 534549.

Morel, G., Malis, E., Boudet, S., 1998. Impedance based combination of visual and force control. In IEEE Int. Conf. on Robotics and Automation, Leuven, Belgium, pp. 1743-1748.

Namiki, A., Nakabo, I., Ishikawa, M., 1999. High speed grasping using visual and force feedback. In IEEE Int Conf. on Robotics and Automation, Detroit, MI, pp. 3195-3200.

Olsson, T., Bengtsson, J., Johansson, R., Malm, H., 2002. Force Control and Visual Servoing Using Planar Surface Identification, In IEEE Int. Conf. on Robotics and Automation. Washington, USA. Pp. 4211-4216.

Pomares, J., Torres, F., 2005. Movement-flow based visual servoing and force control fusion for manipulation tasks in unstructured environments. IEEE Transactions on Systems, Man, and Cybernetics-Part C. Vol. 35, No. 1. Pp. $4-15$.

Tsuji, T., Hiromasa, A., Kaneko, M., 1997. Non-contact impedance control for redundant manipulators using visual information, In IEEE Int. Conf. on Robotics and Automation, Albuquerque, USA. vol. 3, pp. 2571-2576.

Willsky, A. S., Jones, H. L., 1976. A generalized likelihood ration approach to the detection and estimation of jumps in linear systems. IEEE Trans. Automat. Contr., vol. 21, no. 1, pp. 108-112.

Zhang, Z., 2000. A flexible new technique for camera calibration. IEEE Transactions on Pattern Analysis and Machine Intelligence, vol. 22, no. 11, pp. 1330-1334. 